\title{
Arbor
}

\section{Tendencias en la Política de la Educación de la Unión Europea}

\author{
José Antonio Ibáñez-Martín
}

Arbor CLXXIII, 681 (Septiembre 2002), 39-53 pp.

El Tratado de la Unión Europea firmado en Maastricht en 1992 introdujo cambios revolucionarios en las competencias de la Comunidad, pues, por vez primera, se admite que la acción de la Comunidad implicará «una contribución a una enseñanza y a una formación de calidad, así como el desarrollo de las culturas de los Estados miembros». La evolución de esta nueva situación no ha sido satisfactoria por los problemas de fondo de la Unión Europea y por las dificultades que hoy entraña toda acción verdaderamente educativa. El artículo analiza los intentos que se realizaron a partir del 1992 para introducir una profunda dimensión europea de la educación, con las implicaciones axiológicas que ello comporta, y cómo estos intentos se encontraron ante diversos obstáculos poderosos que les han reducido a un estado de hibernación. A partir de fines del 1999 se consolida una nueva tendencia funcionalista en la política de la educación europea, que ha sido refinada en estos dos años, encontrándonos ahora, por mucho que sean limitados sus horizontes, ante unas pautas de trabajo que pienso serán beneficiosas para introducir mayores cotas de racionalidad en la política educativa de los Estados miembros.

\section{La revolución de Maastricht}

Han pasado diez años desde la firma en Maastricht el 7 de febrero de 1992 del Tratado de la Unión Europea, que introdujo cambios revolucio- 
narios en la acción comunitaria dentro del ámbito de la educación y la cultura. En efecto, como es sabido, dicho Tratado, sintiendo «la necesidad de sentar unas bases firmes para la construcción de la futura Europa», «deseando acrecentar la solidaridad entre sus pueblos, dentro del respeto de su historia, de su cultura y de sus tradiciones» y «resueltos a crear una ciudadanía común a los nacionales de sus países» ${ }^{1}$, en el igual respeto a «la identidad nacional de sus Estados miembros» ${ }^{2}$, decide que la Unión Europea adquiera ciertas competencias en el ámbito de la educación -por encima de las que ya tenía en la formación profesional-y en el de la cultura. Concretamente, se determina que la acción de la Comunidad implicará «una contribución a una enseñanza y a una formación de calidad, así como el desarrollo de las culturas de los Estados miembros» ${ }^{3}$, terminología con la que se significa que no nos encontramos, en este asunto, ante un ámbito de competencia exclusiva de la Comunidad, por lo que en él, ésta «intervendrá, conforme al principio de subsidiariedad, sólo en la medida en que los objetivos de la acción pretendida no puedan ser alcanzados de manera suficiente por los Estados miembros, y, por consiguiente, puedan lograrse mejor, debido a la dimensión o a los efectos de la acción contemplada, a nivel comunitario» ${ }^{4}$. La concreta especificación de los objetivos que se plantea el nuevo Tratado en estos ámbitos se encuentra en los artículos 126,127 y 128 , hoy 149,150 y $151^{5}$, en donde también se precisa que para tomar decisiones comunitarias -siempre «en el pleno respeto de sus [de los Estados miembros] responsabilidades en cuanto a los contenidos de la enseñanza y a la organización del sistema educativo, así como de su diversidad cultural y lingüística» ${ }^{6}$ - se seguirán ciertos procedimientos de codecisión o de exigencia de mayorías cualificadas para adoptar medidas de fomento o Recomendaciones.

Ahora bien, realmente, la comparación entre las metas señaladas en lo que hemos llamado Preámbulo del Tratado, su concreta especificación en los artículos citados y las numerosas iniciativas que se han desarrollado basándose en ellos, es bastante descorazonadora, si bien muy expresiva de los problemas de fondo de la Unión Europea y de las dificultades que hoy entraña toda acción verdaderamente educativa.

\section{Una interpretación de las nuevas metas del Tratado de Maastricht desde un punto de vista educativo}

Comencemos reflexionando sobre las nuevas metas. Pienso que es bastante indiscutible que si verdaderamente se quiere sentar unas bases 


\section{Tendencias en la Política de la Educación}

firmes para la construcción de una Europa unida, en la que todos tengan una ciudadanía común, será precisa una reflexión sobre lo que nos une a los europeos -no olvidemos que los intereses económicos son más frecuentemente causa de división que de unidad, precisamente porque no pueden igualmente ser disfrutados por todos-, lo que facilitará la determinación de los grandes objetivos educativos comunes, y lo que ayudará a promover una ciudadanía que no se reduzca al pasivo goce de derechos, sino que se manifieste igualmente en la preocupación por trabajar activamente por el bien de la ciudad que nos acoge y tenemos por propia, también estudiando cuáles son los derechos que debemos establecer y luchando luego para su aprobación y realización efectiva. En pocas palabras: una interpretación pertinente y profunda de las metas señaladas, mueve al pensamiento de que, a partir de la firma del Tratado de Maastricht, la Unión Europea se empeñaría en analizar los elementos básicos de la identidad europea y en movilizar los medios pertinentes para fomentarla, siempre dentro del necesario respeto a las identidades nacionales de los Estados miembros.

Por ello, cuando leemos que «la acción de la Comunidad se encaminará a desarrollar la dimensión europea de la enseñanza» ${ }^{7}$, así como que la Comunidad se esforzará en poner de relieve «el patrimonio cultural común» ${ }^{8}$, cabría pensar que en verdad hemos comenzado una etapa nueva. Más aún, podríamos incluso recordar que tal etapa no se debería calificar, estrictamente, de nueva, pues tiene diversos antecedentes, entre los que citaremos la Resolución del Consejo de 24 de mayo de 1988, cuyo objetivo era: «fortalecer en los jóvenes el sentido de la identidad europea y aclararles el valor de la civilización europea, de las bases sobre las cuales los pueblos europeas pretenden hoy en día fundar su desarrollo, concretamente la salvaguardia de los principios de la democracia, la justicia social y el respeto de los derechos humanos» ${ }^{9}$, para lo que consideraba necesario incluir «la dimensión europea en sus programas escolares en todas las asignaturas pertinentes, por ejemplo, en la literatura, los idiomas, la geografía, las ciencias sociales, la economía y las artes» ${ }^{10}$. Indudablemente era una Resolución ambiciosa, pues conjugaba una llamada a la investigación sobre los fines, con la promoción de los medios pedagógicos operativamente imprescindibles, siendo evidente la necesidad de empeñarse ya desde la escuela en la tarea de superar los prejuicios que existen en todas las naciones europeas sobre los restantes Estados miembros de la Unión y los estereotipos imperantes sobre el carácter de sus ciudadanos. Una propuesta, después del Tratado de Maastricht, acerca de los fines la encontramos, por ejemplo, en el Dictamen del Consejo Económico y Social sobre «La Europa de los Ciudadanos» del 23 de sep- 
tiembre de 1992, donde se afirma que «Una Europa de los Ciudadanos no constituye un 'retrato robot', ni un 'crisol' en el que cabe todo. La clave para una Europa de los Ciudadanos reside en la unidad y la diversidad de sus culturas, su pluralismo de pensamiento y tradiciones, su herencia cristiana y apreciación de otros credos, así como los valores y principios humanísticos y cívicos, y su identificación fundamental con la libertad, la paz, la justicia social, la tolerancia, los derechos humanos y el imperio de la ley. El 'espíritu' de Europa está en efecto impregnado de los principios humanistas -especialmente el derecho de la Persona al respeto de su dignidad-que constituyen la base y el motor de la Democracia. La 'idea' de la Europa de los Ciudadanos se encuentra firmemente enraizada en dichos valores democráticos comunes y en el respeto de la dignidad humana. Estos valores comunes inspiraron la mentalidad europea contemporánea y el concepto de democracia transnacional que, frente a las limitaciones del nacionalismo intransigente, pueden ofrecer un respeto absoluto de la diversidad, de la posibilidad de elección y de la calidad, además de garantizar mejor un entendimiento verdadero y pacífico entre los pueblos» ${ }^{11}$. También cabe recordar que, sobre los medios pedagógicos, hubo un empeño por promover -a partir de esta Resolución- un texto común de historia, materia especialmente importante para facilitar una reconstrucción de la historia europea no basada en las diferencias bélicas que hemos tenido durante siglos sino en los elementos comunes que, como bien reconocían los originarios de otros continentes, nos identificaban como europeos. Así un conocido historiador francés editó, junto con colaboradores diversos, un texto de historia europea, que fue traducido a diversas lenguas para que pudiera usarse en la mayor parte de Europa ${ }^{12}$.

En esta línea -tendencia- que acabamos de señalar, se encuentran también diversos textos no oficiales, de los que voy a referirme solamente a dos. El primero es el informe del Grupo de estudio sobre educación y formación que se nombró por la Comisión en julio de 1995 con la función de requerir a un conjunto de asesores independientes -veintidós personas de los diversos Estados miembros, presididos por el Prof. Jean-Louis Reiffers- para que estudiaran el tipo de educación que exigiría la sociedad del futuro, dándoles un plazo de dos años. Este Grupo emitió su primer informe provisional en 1996, publicándose el 3 de febrero de 1998 el informe final. El análisis de este largo documento es muy interesante por el equilibrio que manifiesta. En efecto, la tesis defendida es que es preciso conjugar el fortalecimiento de la competitividad económica de Europa con el respeto a los principios básicos de la educación «cuyos fines van mucho más allá que los que se descubren desde una perspectiva puramente utilitarista» ${ }^{13}$. Por ello, la nueva ciudadanía europea exige, en 


\section{Tendencias en la Política de la Educación}

primer lugar, «afirmar y transmitir los valores comunes en los que se funda su civilización", valores que el Grupo estuvo de acuerdo en afirmar eran los siguientes:

- derechos humanos/dignidad humana

- libertades fundamentales

- paz y rechazo al uso instrumental de la violencia

- respeto a los demás

- espíritu de solidaridad (dentro de Europa y con el resto del mundo)

- desarrollo equitativo

- igualdad de oportunidades

- los principios del pensamiento racional: la aceptación de la evidencia y la prueba como exigencia ética

- preservación del ecosistema

- responsabilidad personal ${ }^{14}$

Algunos de estos valores aparecen en numerosos documentos. Pero otros no suelen citarse, cuando son muy importantes. Hoy vemos la importancia del rechazo al uso instrumental de la violencia, pero quizá no valoramos la trascendencia que tiene subrayar la responsabilidad personal, tanto por encima de un Estado asistencial que fomentara la desresponsabilización del individuo sobre su propia suerte, como de una mística identitaria de grupo en la que el individuo no pasara de ser un elemento de un engranaje superior; quizá no valoramos suficientemente, debido a la marea del pensamiento débil, los principios del pensamiento racional y la capacidad de comprometer la propia vida según las evidencias que proporciona el entendimiento, olvidándonos que es aquí precisamente donde se encuentra una de las raíces más fecundas de la huella que la civilización europea ha dejado en la historia de la humanidad.

El segundo es un trabajo mío que expuse en una Mesa Redonda sobre la política educativa en la Unión Europea después de Maastricht, para cuya coordinación fui invitado por la Presidencia de la reunión anual de la American Educational Research Association, que se celebró en 1995 en San Francisco, y que luego publiqué en España. Tal trabajo tenía como punto de arranque unas palabras de Ortega y Gasset acerca de la «conciencia cultural europea» ${ }^{15}$, que, según él, se caracterizaba por la aspiración a alcanzar «la manera más perfecta de ser hombre» ${ }^{16}$, y que yo desarrollaba en una interpretación de lo que considero es la identidad específica de la cultura europea, analizando los tres elementos esenciales que caracterizan a una cultura en sentido objetivo, que son el modo como se define al ser humano -y el juego entre lo que recibe de la sociedad y el futuro personal que proyecta-, la forma como se entienden las relaciones 


\section{José Antonio Ibáñez-Martín}

entre los hombres, y el tipo de presencia/ausencia que la divinidad tiene en la vida de la persona y de la sociedad ${ }^{17}$, entrando, por consiguiente, en una reflexión sustancialmente axiológica, cuyos términos exactos no puedo desarrollar aquí.

Ahora bien, los hechos muestran, a todas luces, que esta tendencia ha tenido escasa importancia, hasta el punto que me atrevería a decir que hoy se encuentra en estado de hibernación oficial. Expongamos las causas de esta situación.

\section{Las ambigüedades de un articulado y los problemas de fondo de la Unión Europea en la formulación de una Política de la Educación}

Sería ingenuo creer que hay una aceptación irrestricta al proceso de unión de las distintas naciones de Europa. Casi nadie pone en tela de juicio la conveniencia de arbitrar sistemas que faciliten el crecimiento económico de todos, que proporcionen una imagen colectiva más fuerte, especialmente frente al poderío de los Estados Unidos, que mejoren la defensa, disminuyendo a la vez -gracias a los efectos de sinergia producidos por la unidad de acción- sus costos etc. Pero otra cosa muy distinta es desarrollar políticas que deterioren o comprometan la especificidad e identidad del propio país. Para no pocas personas «el compromiso con el proyecto europeo tiende a ser monopolio, esencialmente, de una amplia clase media de cuadros (y sus hijos) en el ámbito académico, profesional, de negocios o de empleados públicos» ${ }^{18}$, que «contrasta con el sentido de la identidad de los ciudadanos ordinarios», lo que mueve a la idea de que «la emergencia de una identidad europea ampliamente compartida me parece sumamente improbable» ${ }^{19}$.

Las consecuencias de estos planteamientos reticentes las observamos ya en el mismo articulado de Maastricht, en los números dedicados a la educación y a la cultura. En ellos se habla de la «dimensión europea de la educación», concepto que queda inmediatamente aguado cuando se le añade «especialmente a través del aprendizaje y difusión de las lenguas de los Estados miembros» ${ }^{20}$, o cuando se especifica, como gran finalidad, «favorecer la movilidad de estudiantes y profesores» ${ }^{21} \mathrm{o}$ el incremento «de animadores socioeducativos» 22 . Como es obvio, esa cierta esquizofrenia entre metas del Preámbulo y concreciones del articulado, ha llevado a que bastantes autores se hayan limitado a interpretar la "dimensión europea» según mediocres aspiraciones de promover proyectos pedagógi- 


\section{Tendencias en la Política de la Educación}

cos comunes a realizar entre persona de distintos países europeos, animándoles a trabajar en equipo, dentro de unas vagas referencias a la democracia y el interculturalismo ${ }^{23}$, lo que, por otra parte, venía a ser sustanciosamente apoyado por los programas comunitarios de mayor dotación económica.

Pero, a todo ello, es preciso sumar los problemas que actualmente surgen cuando alguien desea empeñarse en una acción auténticamente educativa, impregnada esencialmente de aquellos valores que se consideran configuran el escenario de nuestra plenitud humana. No tengo duda alguna que esa es una empresa epistemológicamente complicada, como vemos ya en el caso de Ortega: es significativo que el manuscrito de la conferencia citada nunca llegó a acabarse, en el fondo porque a pesar de los clamores de numerosos intelectuales tras la Primera Gran Guerra -y cuánto más tras los horrores de la Segunda Guerra Mundial-pidiendo un cambio en los ideales sociales ${ }^{24}$, que sustituyeran el eficiencismo y el afán de dominio por un nuevo ideal de respeto a la dignidad de la persona, de solidaridad y de servicio, no ha habido vigor social y argumentativo para que se impusiera un nuevo paradigma axiológico, por lo que el ambiente espiritual de nuestros días, como diría Jaspers, es de considerable indigencia.

Obviamente, el conjunto de estos problemas ha tenido unas consecuencias políticas claras, pues quienes se encontraban en los órganos de gobierno comunitarios eran conscientes del peligro de naufragio de la Unión Europea -por mucho que se acudiera al principio de subsidiariedad-si se pretendiera involucrarla realmente en el ámbito educativo y cultural, si se quisiera, por ejemplo, orientar la actividad comunitaria a promover esa común identidad que, usando las citadas palabras de Ortega, expresara esa «manera más perfecta de ser hombre» que defendemos los europeos. La conclusión de todos estos problemas ha sido doble: por una parte la tendencia anteriormente descrita fue hibernada, y, por otro lado, la política de la educación europea ha ido dando tumbos durante largos años, careciendo de un norte claro. Pero como no hay mal que cien años dure, creo que el horizonte hoy ha cambiado, habiéndose producido una mayor reflexión sobre lo que la Unión debería hacer en el ámbito de la educación y la formación -teniendo también en cuenta las aportaciones del documento titulado «Agenda 2000»- y lo que realmente podía hacer, considerando las dificultades que acabamos de exponer y considerando igualmente que la Unión sólo está llamada a proporcionar una contribución, siendo los Estados miembros quienes tienen la responsabilidad de facilitar a sus ciudadanos una educación realmente integral. 


\section{Las nuevas tendencias en la Política de la Educación europea}

Tal cambio se inicia con la Resolución del Consejo de 17 de diciembre de 1999 «Hacia el nuevo milenio»: elaboración de nuevos procedimientos de trabajo para la cooperación europea en el terreno de la educación y la formación. En el Preámbulo de esta Resolución, leemos que el objetivo buscado es organizar el trabajo del Consejo en el terreno de la educación y la formación según un programa continuado -es decir, determinar una auténtica política, que exige el mantenimiento de determinados empeños a lo largo del tiempo- que durara varias Presidencias que buscara atender ciertos «asuntos prioritarios (que) se tratarían de un modo cíclico» ${ }^{25}$, destacándose que «el principal objetivo de crear un marco de este tipo es dar mayor continuidad, eficacia, eficiencia y fuerza a los efectos políticos de la cooperación comunitaria en el terreno de la educación y la formación. El nuevo marco de cooperación permitiría un intercambio más eficaz de la información y las buenas prácticas» ${ }^{26}$. Al final de la Resolución, se precisa que los asuntos prioritarios, para empezar, serán: «papel de la educación y la formación en las políticas de empleo; desarrollo de la calidad de la educación y la formación, en todos los niveles, y fomento de la movilidad, incluido el reconocimiento de las cualificaciones y las temporadas dedicadas al estudio» ${ }^{27}$.

Considero que esta decisión, aun limitada en sus pretensiones, ha sido altamente clarificadora, pues desde este momento se han ido tomando decisiones concretas, que han incidido sobre las prioridades del 17 de diciembre de 1999, y que muestran una profundidad hasta ahora desconocida en el empeño por formular una auténtica política de la educación.

La primera reunión del Consejo Europeo que se mantuvo tras la Resolución citada fue en Lisboa, en marzo de 2000. En las Conclusiones del Consejo leemos que «el nuevo objetivo estratégico para la próxima década: convertirse en la economía basada en el conocimiento más competitiva y dinámica del mundo, capaz de crecer de manera sostenible con más y mejores empleos y con mayor cohesión social» ${ }^{28}$, lo que se desarrolla en una petición al Consejo de Educación para «que emprenda un reflexión general sobre los futuros objetivos precisos de los sistemas educativos, centrada en intereses y prioridades comunes y que respete al mismo tiempo la diversidad nacional» ${ }^{29}$. Tal reflexión se produjo en un Informe de la Comisión de fecha 31 de enero de $2001^{30}$ que, tras analizar las respuestas de los Estados miembros, concretaba determinados objetivos -que en el punto 7 dice que son cinco, pero que, en realidad, terminan siendo seis- que se consideran podrían ser la base de la colaboración eu- 


\section{Tendencias en la Política de la Educación}

ropea en los próximos diez años. Estos objetivos son: mejorar la calidad en Europa, facilitar y ampliar el acceso al aprendizaje a cualquier edad, actualizar la definición de capacidades básicas de acuerdo con la sociedad del conocimiento, abrir la educación y la formación al entorno local, a Europa y al mundo (obsérvese que abandonamos la dimensión europea), aprovechar al máximo los recursos, y crear nuevas relaciones con los centros escolares.

Estos grandes objetivos son oportunamente desglosados y así se considera que:

1) Mejorar la calidad del aprendizaje significa: a) mejorar la formación de profesores y formadores, teniendo en cuenta que ya no son la única fuente de conocimientos, pero que deberían motivar a sus : alumnos no sólo para que aprendan las capacidades profesionales necesarias sino para responsabilizarse también de su propio camino; b) aumentar la alfabetización y la formación aritmética elemental, base para poder acceder al empleo y que no pocos pierden una vez abandonan el contexto del aprendizaje formal.

2) Facilitar y ampliar el acceso al aprendizaje a cualquier edad implica: a) que los sistemas educativos se adapten a las necesidades de las personas de todas las edades y sepan atraer a gente de cualquier edad, lo que pienso es una nueva reflexión actualizada sobre las implicaciones del derecho a la educación, que no debe reducirse a un rígido marco de edad, procurando que el sistema educativo se muestre acogedor con estudiantes de circunstancias muy diversas; b) hacer más atrayente el aprendizaje, de forma que se contrarreste el aliciente que muchos jóvenes -en mi experiencia, especialmente los varones- encuentran en incorporarse tempranamente a un trabajo en busca de una remuneración que les proporcione una cierta independencia; c) coherencia interna de los sistemas educativos, con flexibilidad y facilidad de cambiar las trayectorias formativas, permitiendo el acceso a la enseñanza superior desde formaciones muy distintas; d) educación como medio de cohesión social, adaptándose a las necesidades de los diversos grupos sociales y transmitiendo a través de los planes de estudio y de los materiales de la enseñanza la imagen de la sociedad a la que aspiramos, es decir la sociedad en la que reine la igualdad de oportunidades y se haya desterrado el racismo y la discriminación.

Este conjunto de objetivos considero debiera ser especialmente atendido en España, donde por muchos años estamos sometidos al dogma del currículo único, al imperio del bachillerato como único 
medio ordinario para acceder a la Universidad y a la obsesión por creer que la formación profesional tendrá calidad y reconocimiento social endureciendo las exigencias académicas para acudir a ella. Confiemos en que los actuales proyectos de reformas en nuestro sistema educativo nos permitan salir de esos hechizos.

3) Actualizar la definición de capacidades básicas de acuerdo con la sociedad del conocimiento, que debe comprender tanto las de carácter profesional como las de carácter personal o social, que permiten trabajar con los demás y llevar una vida feliz y fructífera. Así se considera necesario: a) poner las tecnologías de la información y la comunicación al alcance de todos; y b) revisar el significado de las capacidades básicas, conscientes de la importancia de capacitar para el aprendizaje autónomo, pero también capacitar para la tolerancia con los demás y con la autoridad, para adaptarse y trabajar en equipo, resolver problemas y asumir riesgos etc.

4) Abrir la educación y la formación al entorno local, a Europa y al mundo, lo que exige a) una mejor enseñanza de los idiomas, con presencia de profesores de idiomas nativos; b) aumento de la movilidad y los intercambios, ampliando la gama de centros escolares que participan en los programas europeos, donde las instituciones con alumnos menos favorecidos tienden a estar infrarrepresentadas; c) intensificación de las relaciones con las empresas, cambiando tradicionales actitudes de protección de la escuela contra las influencias externas, para descubrir qué lecciones se pueden extraer de la participación del sector empresarial; d) desarrollo del espíritu de empresa, como espíritu activo y reactivo que la sociedad debiera valorar y apoyar.

5) Aprovechar al máximo los recursos, lo que obliga a: a) establecer sistemas que aseguren la calidad para que el sistema funcione eficazmente y que faciliten la publicidad de sus resultados, aumentándose así la confianza social sobre los centros escolares; b) adaptar los recursos a las necesidades reales, lo que exige que las autoridades competentes conozcan lo que va bien y lo que va mal, de modo que se orienten los recursos en las direcciones apropiadas, lo que sin duda alguna disgustará a todos aquellos que temen que la calidad sea reconocida y valorada.

6) Crear nuevas relaciones de la autoridad con los centros escolares, sin obligar a que en todos se tenga el mismo grado de intervención, que debiera depender del éxito de la institución, sin que se cuestionara la actuación de los directores de los centros que tuvieran resultados brillantes. 


\section{Tendencias en la Política de la Educación}

El siguiente documento de interés es la Comunicación de la Comisión de 21 de noviembre de 2001 titulada «Hacer realidad un espacio europeo del aprendizaje permanente», fruto de la consulta a más de 12.000 personas sobre las exigencias del objetivo 2), previamente señalado. Es también un documento largo, que señala seis prioridades de actuación a la hora de actuar con estrategias coherentes y globales de aprendizaje permanente.

Ahora bien, considero que el trabajo de mayor relevancia que se ha hecho, y cuya importancia iremos viendo en los próximos años, es el Programa de trabajo para el seguimiento de los objetivos de educación y de los sistemas de formación europeos, de 20 de febrero de $2002^{31}$, presentado al Consejo Europeo de Barcelona, de marzo de 2002. En este documento, tras una reflexión sobre el conjunto de prioridades que se han venido manejando desde el 17 de diciembre de 1999, el acuerdo es que los objetivos estratégicos en educación y sistemas de formación sean los tres siguientes:

1) Mejorar la calidad y eficacia de la educación y de los sistemas de formación en la Unión.

2) Facilitar el acceso a todos a la educación y a los sistemas de formación.

3) Abrir la educación y los sistemas de formación a un mundo más amplio.

La diferencia fundamental con las prioridades del 1999, consiste en que entonces la primera se refería al papel de la educación en las políticas de empleo, lo que suscitó considerables críticas por parte de quienes -como vimos en las conclusiones del Grupo de estudios- entendían que la educación no podía enfocarse desde el punto de vista del empleo, pues se desvirtuaba su esencia e incluso se comprometía su eficacia a largo plazo. Por ello, el término empleo no aparece entre los grandes objetivos, aunque no se encuentra ajeno a algunos de los trece objetivos asociados, que se detallan. Pero, además, el documento señala que, en beneficio de los ciudadanos hay cinco criterios generales de actuación cuya operatividad debiera alcanzarse antes del 2010 y que son:

1) Conseguir la más alta calidad en educación y en formación, de forma que Europa sea reconocida como un punto de referencia mundial por la calidad y relevancia de sus sistemas e instituciones.

2) Hacer compatibles suficientemente la educación y los sistemas de formación, de modo que los ciudadanos pueden moverse entre ellos y obtener las ventajas de su diversidad.

3) Establecer convalidaciones efectivas de las cualificaciones, conocimientos y habilidades que se hayan adquirido en cualquier país de 
la Unión, con el fin de establecer una carrera y un aprendizaje continuo.

4) Facilitar el acceso al aprendizaje permanente a los europeos de todas la edades.

5) Abrir Europa a la cooperación con todas las otras regiones, para beneficio mutuo, de modo que sea el destino más solicitado de los estudiantes, profesores e investigadores de todo el resto del mundo.

El conjunto de este documento -que sólo está en versión inglesa- no puede ser descrito en este artículo, ya que tiene unos cincuenta folios de extensión. Pero sí me parece conveniente señalar que su principal novedad no radica tanto en sus principales objetivos, que expresan un nuevo acercamiento, algo diverso a los ya señalados en el citado Informe de la Comisión de fecha 31 de enero de 2001, cuanto en el esfuerzo que realiza por determinar indicadores cuantitativos de los objetivos, fechas del seguimiento desde la Comisión y temas que pueden ser motivo de intercambio de experiencias y de buenas prácticas. Pongamos un ejemplo. El primer objetivo estratégico -mejorar la calidad y eficacia de la educación y de los sistemas de formación en la Unión- tiene cinco objetivos asociados, de los cuales el segundo es desarrollar las habilidades precisas en la sociedad del conocimiento, que se desglosa en tres elementos centrales en los que se establece la necesidad no de sólo identificarlas sino también de determinar cómo se pueden promover mediante el currículo escolar, unidas a las restantes habilidades básicas, y cómo se pueden mantener a lo largo de la vida. Pues bien, este conjunto de objetivos se traduce en una serie de indicadores, esencialmente cuantitativos, que deben expresar el progreso que se va consiguiendo en su realización, y que en este caso son: personas que completan la enseñanza secundaria, formación permanente de profesores en el ámbito de las habilidades emergentes necesarias, niveles de resultados en las ciencias humanas y sociales (PISA, evaluación realizada en los países miembros, cuyos resultados primeros se dieron a conocer en noviembre de 2001), niveles de resultados en las ciencias exactas y naturales (PISA), aprendizaje para progresar en los niveles de resultados y porcentaje de alumnos que no han llegado a la enseñanza secundaria última y que han participado en alguna forma de enseñanza de adultos, según grupos de edades. Cada país deberá realizar los estudios pertinentes, según estos criterios, de lo que ha ocurrido cada año, pero, además, se precisan algunos temas para intercambiar experiencias y buenas prácticas, que en este objetivo asociado son, concretamente, los modos de actuar y los resultados conseguidos en la enseñanza de la lengua materna, de las lenguas extranjeras y de las matemáticas, 


\section{Tendencias en la Política de la Educación}

hasta la finalización de la enseñanza obligatoria, así como los medios propuestos para conseguir la alfabetización funcional tanto en la escuela como para los adultos.

\section{Conclusiones}

Es evidente que, en la medida en que no se quiere limitar el proyecto de la Unión a configurar una Europa de los mercaderes, es preciso comprometerse en una actividad educativa y cultural común, como se deseó establecer en Maastricht. Ese compromiso tiene una traducción axiológica indeclinable, y en tal sentido se orientaron diversos esfuerzos a partir del 1992. Desgraciadamente, la extendida oposición de fondo a una real unidad europea, sumada a los problemas que hoy entraña embarcarse en una acción educativa auténtica, llevaron a hibernar esos propósitos, que han sido cambiados, a partir del 2000, por otros de carácter funcionalista, depositándose en los Estados miembros la responsabilidad de la necesaria orientación axiológica del sistema educativo. Estos dos últimos años han visto un refinamiento de los primeros objetivos marcados a fines del 1999, y, por mucho que sean limitados en sus horizontes, han marcado unas pautas de trabajo que pienso serán beneficiosas para introducir mayores cotas de racionalidad en la política educativa de los Estados miembros.

\section{Notas} bulo.

1 Tratado de Maastricht, Declaraciones iniciales, sin título, que llamaremos Preám-

2 Ídem, art. F, pár. 3, hoy art. 6.

3 Ídem, art. 3, p), hoy art. 3, q) del Tratado de Roma.

4 Ídem, art. 3. b, hoy art. 5 del Tratado de Roma.

${ }^{5}$ Como es sabido, el Tratado de Ámsterdam del 2 de octubre de 1997 aprobó unos cuadros de equivalencias de los nuevos preceptos sucesivamente aprobados para adscribir una nueva numeración en las versiones consolidadas de los Tratados. A partir de ahora nos referiremos sólo a la numeración de esta última versión.

6 Tratado de Roma, art. 149, pár. 1.

7 Ídem, pár. 2.

8 Ídem, art. 150, pár. 1.

${ }^{9}$ Resolución del Consejo y de los Ministros de Educación reunidos en el «Consejo sobre la dimensión europea en la enseñanza» de 24 de mayo de 1988 (88/C 177/02), I. Objetivos. Aquí cita la Declaración de Copenhague, de 8 de abril de 1978. Esta cita es poco afortunada. El documento en cuestión es la "Declaración sobre la democracia" del Con- 
sejo Europeo de Copenhague, en el que los jefes de Estado o de Gobierno confirman su voluntad, expresada en la Declaración de Copenhague sobre la identidad europea, de garantizar los principios de la democracia representativa etc. Por tanto, pienso que debiera haberse citado lo que suele llamarse la Declaración de Copenhague del 15 de diciembre de 1973, que es, sin duda, el documento de más alto nivel que trata sobre la identidad europea. En esa Declaración, el Anexo 2 al capítulo II se titula «De la identidad europea", donde leemos párrafos tan sustanciosos como los siguientes: «Deseosos de asegurar el respeto de los valores de orden jurídico, político y moral a los que se sienten apegados, preocupados por preservar la rica variedad de sus culturas nacionales, compartiendo una misma concepción de la vida, fundada en la voluntad de construir una sociedad concebida y realizada al servicio de los hombres, esperan salvaguardar los principios de la democracia representativa, del imperio de la ley, de la justicia social -finalidad del progreso económico- y del respeto de los derechos del hombre, los cuales constituyen elementos fundamentales de la identidad europea" (I, 1). Más adelante leemos: «Esta variedad de culturas en el marco de una misma civilización europea, este apegamiento a valores y principios comunes, este paralelismo de las concepciones de la vida, esta conciencia de poseer en común intereses específicos y esta determinación de participar en la construcción europea, le dan a la identidad europea su carácter original y su dinamismo propio" (I, 3).

10 Resolución del Consejo y de los Ministros de Educación reunidos en el «Consejo sobre la dimensión europea en la enseñanza" de 24 de mayo de 1988 (88/C 177/02), II Acción A.

11 Dictamen del Consejo Económico y Social sobre «La Europa de los Ciudadanos» del 23 de septiembre de 1992 CES 1037/92, Apartados 1.2.2. y 1.2.2.

12 DELOUCHE, F.: Das Europäische Geschichtsbuch: von den Anfängen bis heute / eine europäische Initiative. Este libro, como digo, se ha editado y reeditado en diversas lenguas, durante bastantes años, desde el 1992. La edición que cito se publicó en Stuttgart en 1998.

13 Study Group on Education and Training. «Report Accomplishing Europe through Education and Training», DG XXII, de 3 de febrero de 1998, Executive Summary.

14 Ídem $\mathrm{n}^{\circ} 52$.

15 ORTEGA Y GASSET, J. (1985): «Hay hoy una conciencia cultural europea?». en Europa y la idea de nación, 21. Madrid: Revista de Occidente.

16 Ídem, De nación a provincia de Europa, 16, op.cit.

17 IBÁÑ̃ZZMARTÍN, J. A. (1997): “¿Hay una cultura europea tras la política educativa comunitaria? Is there a European Culture behind the European Community Educative policy?». En SANTOS REGO, M. A. (ed.): Politica educativa en la Unión Europea después de Maastricht. Educational policy in the European Union after Maastricht. Santiago de Compostela: EGAP, pp. 17-44. El libro está publicado en español y en inglés, a dos columnas.

18 WILLIAMS, K. (2000): «Realism, Rationalism, and the European Project». En CRAWLEY, F., SMEYERS, P. y STANDISH, P. (eds.): Universities remembering Europe: Nation, Culture and Higher Education. New York-Oxford: Berghahn Books, p. 53.

19 Ídem, 58.

20 Art. 149 , pár. 2.

21 Ídem.

22 Ídem.

23 Exponente y principal causa de esas deficiencias es el Libro Verde sobre la Dimensión Europea de la Educación, de 29 de septiembre de 1993, COM (93) 457 final, en 


\section{Tendencias en la Política de la Educación}

el que se basan numerosos estudios, que no citaré, irrelevantes y confusos, que se han editado bajo esa bandera. El 23 de noviembre de 2000 fui convocado como Experto a una audiencia en el Comité Económico y Social, en Bruselas, para evaluar un documento que estaban preparando sobre la Dimensión Europea de la Educación: naturaleza, contenido y perspectivas. El documento era tan malo como el Libro Verde, y allí defendí la posición de que tratar sobre ese asunto exigía, al menos, tocar cuatro aspectos: los elementos básicos de la identidad cultural europea, sin limitarse a vaciedades y generalidades, los derechos y deberes de los ciudadanos europeos, cómo comprometerse en el mutuo conocimiento entre los jóvenes europeos, superando el turismo ilustrado que propiciaba ahora el Programa Erasmus y pasando a convertirse en una actividad realmente formativa dentro de un itinerario curricular propio europeo, más algunas propuestas imaginativas que, dentro del principio de subsidiariedad, colaboraran en el desarrollo de tal dimensión europea. Un repaso a las consecuencias de las nuevas tendencias de la política de la educación europea me lleva a la conclusión de que se ha ido dejando en una discreta penumbra tal dimensión europea, al haberse, lamentablemente, diseñado como un constructo lleno de contradicciones

24 En el mismo año de 1936 aparecieron dos importantes libros que expresan tales preocupaciones, que son el BERDIAEF, N.: Cinq Méditations sur l'existence. Paris: Montaigne; y STEINBÜCHEL, T.: Der Umbruch des Denkens. Regensburg: Postet.

25 Resolución del Consejo de 17 de diciembre de 1999 «Hacia el nuevo milenio»: elaboración de nuevos procedimientos de trabajo para la cooperación europea en el terreno de la educación y la formación, $(2000 / \mathrm{C} 8 / 04) \mathrm{n}^{\circ} 5$.

26 Ídem, $\mathrm{n}^{\circ} 6$.

27 Idem, Anexo.

28 Conclusiones de la Presidencia del Consejo Europeo de Lisboa, 23 y 24 de marzo de $2000, n^{\circ} 5$.

29 Ídem, $\mathrm{n}^{\circ} 27$.

30 Informe de la Comisión de fecha 31 de enero de 2001: «Futuros objetivos precisos de los sistemas educativos", COM (2001) 59 final.

31 "Detailed work programme on the follow-up of the objectives of education and training systems in Europe», 20 February 2002, n 6365/02. 